\title{
A GAME-THEORETIC FRAMEWORK FOR MULTI-USER MULTIMEDIA RATE ALLOCATION
}

\author{
Yan Chen, Beibei Wang, and K. J. Ray Liu
}

\begin{abstract}
How to efficiently and fairly allocate data rate among different users is a key problem in the field of multiuser multimedia communication. However, most of the existing optimization-based methods, such as minimizing the weighted sum of the distortions or maximizing the weighted sum of the PSNRs, have their weights heuristically determined. Moreover, those approaches mainly focus on the efficiency issue while ignoring the fairness issue. In this paper, we address this problem by proposing a game-theoretic framework, in which the utility/payoff function of each user/player is jointly determined by the characteristic of the transmitted video sequence and the allocated bitrate. We show that with the proportional fairness criterion, the game has a unique Nash equilibrium, according to which the controller can efficiently and fairly allocate the available network bandwidth to the users. Finally, we show several experimental results on real video data to verify the proposed method.
\end{abstract}

Index Terms - Rate allocation, game theory, Nash equilibrium, proportional fairness.

\section{INTRODUCTION}

Nowadays, due to the explosive growth of the Internet and the success of the compression technologies, delay-sensitive multimedia networking applications such as multimedia streaming and multicamera surveillance become more and more popular. Therefore, a fundamental problem in these applications, how to fairly and efficiently allocate the rate among different users, draws great attention.

Rate allocation for a single user has been well investigated in the literature [1]. In single-user rate allocation, the task of the rate manager is to assign the available rate to each frame and each macroblock (MB) to achieve the maximal visual quality. If the channel is shared by multiple users, besides considering the rate allocation within each user (i.e., frame-level rate allocation and MB-level rate allocation), the rate controller needs to consider the rate allocation among users, i.e., user-level rate allocation. A simple user-level rate allocation is the constant bit-rate allocation (CBR), where the available network bandwidth is equally assigned to each user. A major problem of CBR is that it does not consider the characteristic of the video sequences. One way to overcome this disadvantage is to optimize a global objective function that involves the characteristic of all video sequences, e.g., minimize the weighted sum of the distortions or maximize the weighted sum of the PSNRs. However, the solution to such methods is highly related to the weights, which are usually heuristically determined, e.g. uniform weights [2]. Moreover, those methods mainly focus on the efficiency issue. The fairness issue has been

Yan Chen, Beibei Wang and K. J. Ray Liu are with Dept. ECE, University of Maryland, College Park. Email: \{yan, bebewang, kjrliu\}@umd.edu.

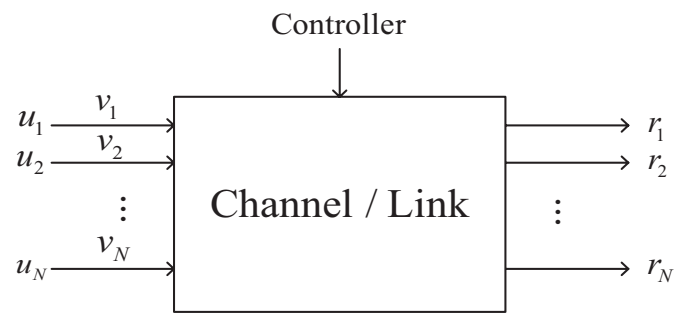

Fig. 1: System Model.

generally ignored. In this paper, we propose a game-theoretic approach to efficiently and fairly allocate the available network bandwidth to different users. Specifically, the utility/payoff function for each user/player is defined according to the characteristic of the transmitted video sequence and the allocated bitrate. Then, we discuss the Nash equilibrium of the rate allocation game. We show that with the proportional fairness criterion, a unique Nash equilibrium can be determined, based on which the rate controller can efficiently and fairly allocate the available rate. We also show several experimental results on real video data to demonstrate the efficiency and effectiveness of the proposed method.

The rest of this paper is organized as follows. In section II, we give a detailed description on the proposed method. In Section III, we first show the detailed processes of estimating the parameters used in the proposed method. Then, we illustrate the experimental results on real video signals. Finally, conclusions are drawn in Section IV.

\section{THE PROPOSED METHOD}

\subsection{Video Distortion-Rate Model}

In video compression, there exists a tradeoff between the distortion $D$ and bit-rate $R$ due to quantization. High bit-rate leads to small distortion while low bit-rate causes large distortion. In the literature, several models have been proposed to characterize this tradeoff for different video coders, such as H.263 [3] and H.264 [4]. Without loss of generality, here we use a simple twoparameter model, which is widely employed in a medium or high bit-rate situation, and other models can be similarly analyzed. The two-parameter distortion-rate model is described as follows:

$$
D(R)=\alpha \exp ^{-\beta R},
$$

where $\alpha$ and $\beta$ are two positive parameters determined by the characteristic of the video content.

\subsection{User's Utility Function}

As shown in Figure 1, user $u_{i}$ can get gain by successfully transmitting the video $v_{i}$ to receiver $r_{i}$, and the gain is determined by the quality of the transmitted video. On the other hand, user $u_{i}$ 
needs to pay for the used bandwidth to transmit $v_{i}$, and the payment is determined by the bit-rate of $v_{i}$. Therefore, the utility function of user $u_{i}$ can be defined as:

$$
U_{i}\left(R_{i}, D_{i}\right)=f\left(D_{i}\right)-a g\left(R_{i}\right)
$$

where $f\left(D_{i}\right)$ is the gain, $g\left(R_{i}\right)$ is the cost, and $a$ is a parameter controlling the balance between the gain and the cost. Without loss of generality, we assume that the cost per bit-rate unit is one, which means:

$$
g\left(R_{i}\right)=R_{i}
$$

In general, $f\left(D_{i}\right)$ is determined by how much $r_{i}$ is satisfied with the received video. In video processing community, the peak signal-to-noise ratio (PSNR) is a more common objective quality measure than MSE. For any MSE $D$, the corresponding PSNR is given by $P S N R=10 \log _{10} \frac{255^{2}}{D}$. Moreover, the quality difference in the low PSNR region is easier to be distinguished than that in the high PSNR region. Therefore, we defined the $f($.$) as:$

$$
f\left(D_{i}\right)=\ln \left(P S N R_{i}\right)=\ln \left[10 \log _{10} \frac{255^{2}}{D_{i}}\right] .
$$

Combining Eqn. (1-4) and ignoring the constant term, the utility function of user $u_{i}$ becomes:

$$
U_{i}\left(R_{i}\right)=\ln \left(\gamma_{i}+\beta_{i} R_{i}\right)-a R_{i}
$$

where $\gamma_{i}=2 \ln 255-\ln \alpha_{i}$.

\subsection{Problem Formulation}

As shown in Figure 1, in this game, there are $N$ users, which cooperate with each other to divide the available network bandwidth. Each user $u_{i}$ has its own utility function as shown in Eqn. (5), and it also has a minimum desired quality constraint (minimal rate constraint $R_{i}^{\text {min }}$ ) and a maximum satisfied quality constraint (maximum rate constraint $R_{i}^{\max }$ ). Since $R_{i}^{\min }$ is the minimal rate constraint that each user expects by jointing the game without cooperation, we assume that the available network rate at least guarantees each user for the minimal desired rate in the cooperative game. Obviously, if the available network bandwidth is able to satisfy all the user with the maximum quality constraint $R_{i}^{\max }$, the rate allocation problem is trivial since the controller just needs to allocate $R_{i}^{\max }$ to each user $u_{i}$. However, in the case that the available network bandwidth is not enough to satisfy all the user with $R_{i}^{\max }$, the problem becomes more interesting: how does the controller fairly and efficiently allocate the available bandwidth to the users? From the users' point of view, they try to maximize their utilities subject to the constraint that the sum of the users' bit-rate does not exceed the available bandwidth. Therefore, the game can be formulated as:

$$
\begin{array}{cl}
\max _{R_{i}} & U_{i}\left(R_{i}\right)=\ln \left(\gamma_{i}+\beta_{i} R_{i}\right)-a R_{i}, \\
\text { s.t. } & R_{i}^{\text {min }} \leq R_{i} \leq R_{i}^{\text {max }}, \quad \forall i=1,2, \ldots, N .
\end{array}
$$

with the global constraint: $\sum_{i=1}^{N} R_{i} \leq R$, where $R$ is the available network bandwidth.

\subsection{Analysis of The Proposed Game}

According to Eqn. (5), we can see that the utility function $U_{i}\left(R_{i}\right)$ is a concave function in term of $R_{i}$. By taking the derivative of $U_{i}\left(R_{i}\right)$ over $R_{i}$, we have:

$$
\frac{\partial U_{i}\left(R_{i}\right)}{\partial R_{i}}=\frac{\beta_{i}}{\gamma_{i}+\beta_{i} R_{i}}-a, \quad \forall i=1,2, \ldots, N .
$$

Therefore, user $u_{i}$ achieves its maximal utility $U_{i}^{\star}\left(R_{i}^{\star}\right)$ at $R_{i}^{\star}$, where $R_{i}^{\star}$ is defined as:

$$
R_{i}^{\star}=\max \left[R_{i}^{\min }, \min \left(\frac{1}{a}-\frac{\gamma_{i}}{\beta_{i}}, R_{i}^{\max }\right)\right], \forall i .
$$

From Eqn. (8), we can see that the optimal $R_{i}^{\star}$ corresponding to the maximal utility is determined by the parameter $a$. Therefore, for different choices of $a$, the game in Eqn. (6) has different equilibria with different physical meanings. Let $a^{0}$ be the constant such that $\sum_{i=1}^{N}\left(\max \left[R_{i}^{\min }, \min \left(\frac{1}{a^{0}}-\frac{\gamma_{i}}{\beta_{i}}, R_{i}^{\max }\right)\right]\right)=R$. Specifically, in the following, we discuss three different cases: $0 \leq a<a^{0}, a=a^{0}$, and $a>a^{0}$, and analyze the corresponding Nash equilibrium (NE).

1) If $0 \leq a<a^{0}$, the game has infinitely many efficient NE. To choose one reasonable NE $\left(\tilde{R}_{1}, \tilde{R}_{2}, \ldots, \tilde{R}_{N}\right)$, we adopt the proportional fairness criterion, and the proportionally fair NE can be obtained by solving the following optimization problem [5]:

$$
\begin{array}{ll}
\max _{R_{i}} & \sum_{i=1}^{N} \ln \left[\ln \left(\gamma_{i}+\beta_{i} R_{i}\right)-a R_{i}\right] \\
\text { s.t. } & R_{i}^{\text {min }} \leq R_{i} \leq R_{i}^{\text {max }}, \quad \forall i=1,2, \ldots, N ; \\
& \sum_{i=1}^{N} R_{i} \leq R .
\end{array}
$$

Since $\ln \left[\ln \left(\gamma_{i}+\beta_{i} R_{i}\right)-a R_{i}\right]$ is concave in terms of $R_{i}$, the above optimization problem is convex. Therefore, we are able to find the optimal solution using numerical methods.

2) If $a=a^{0}$, the game has a unique NE $\left(R_{1}^{\star}, R_{2}^{\star}, \ldots, R_{N}^{\star}\right)$. Since $a=a^{0}, \sum_{i=1}^{N} R_{i}^{\star}=R$, which means that the available network bandwidth is fully utilized. Moreover, by using KKT conditions, we can prove that this NE is the solution to the following optimization problem:

$$
\begin{aligned}
\max _{R_{i}} & \prod_{i=1}^{N} P S N R_{i} \\
\text { s.t. } & R_{i}^{\text {min }} \leq R_{i} \leq R_{i}^{\text {max }}, \forall i=1,2, \ldots, N ; \\
& \sum_{i=1}^{N} R_{i} \leq R .
\end{aligned}
$$

Therefore, this optimal solution is the proportionally fair NE in terms of both PSNR and utility.

3) If $a>a^{0}$, the game has a unique NE $\left(R_{1}^{\star}, R_{2}^{\star}, \ldots, R_{N}^{\star}\right)$. Since $a>a^{0}, \sum_{i=1}^{N} R_{i}^{\star}<R$, which means that the available network bandwidth is not fully utilized. Therefore, this allocation scheme is not efficient.

\section{EXPERIMENTAL RESULTS}

In order to evaluate the proposed game-theoretic multi-user rate allocation model, we conduct the experiments on real video data. 

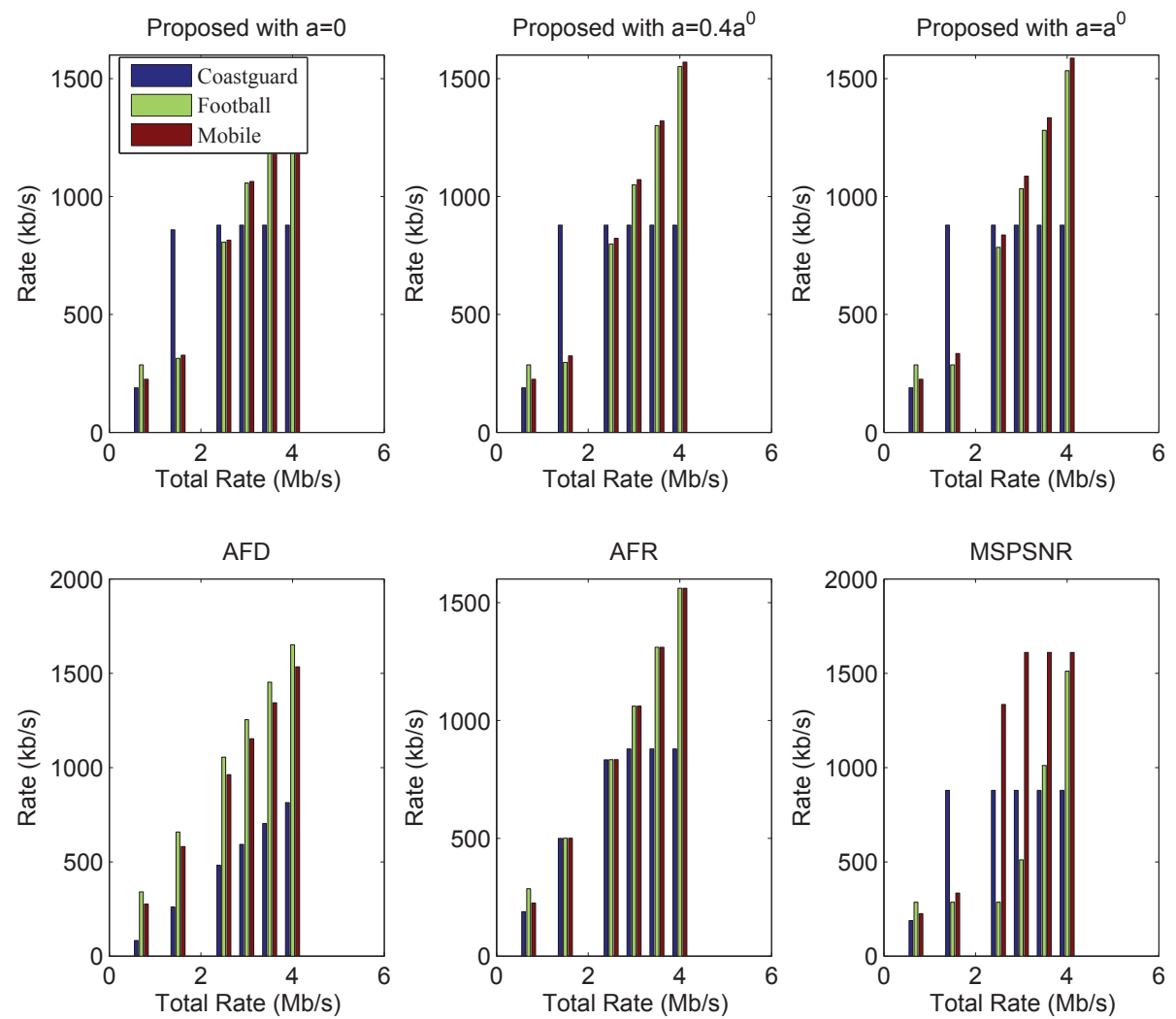

Fig. 2: Allocated Rates For Coastguard, Football and Mobile Using Different Methods.

Table 1: $\gamma_{i}^{\star}, \beta_{i}^{\star}, R_{i}^{\min }(k b / s)$, and $R_{i}^{\max }(k b / s)$ by training.

\begin{tabular}{|c|c|c|c|c|}
\hline Sequence & $\gamma_{i}^{\star}$ & $\beta_{i}^{\star}$ & $R_{i}^{\min }$ & $R_{i}^{\max }$ \\
\hline Coastguard & 6.68 & 0.0043 & 28.5 & 878.8 \\
\hline Football & 6.22 & 0.0024 & 286.3 & 1720 \\
\hline Mobile & 6.35 & 0.0025 & 225.1 & 1610 \\
\hline
\end{tabular}

Three video sequences: mobile, coastguard, and football in QCIF format, are tested. We use the state-of-art H.264 JM 9.0 video codec to encode the video sequences. By changing the quantization parameter (QP) or using the rate control feature, we are able to compress the video sequences at different bit-rates and achieve different quality requirements.

\subsection{Parameter Estimation}

From Section II, we can see that there are several parameters in our framework, $\gamma_{i}, \beta_{i}, R_{i}^{\min }, R_{i}^{\max }$, and $a$. In this subsection, we will discuss how to estimate these parameters.

According to Eqn. (1), we have $P S N R_{i}=\left(10 \log _{10} e\right)\left(\gamma_{i}+\right.$ $\left.\beta_{i} R_{i}\right)$. Therefore, $\gamma_{i}$ and $\beta_{i}$ can be estimated using off-line training:

$$
\left(\gamma_{i}^{\star}, \beta_{i}^{\star}\right)=\min _{\gamma_{i}, \beta_{i}} \sum_{j}\left[P S N R_{i}(j)-10\left(\log _{10} e\right)\left(\gamma_{i}+\beta_{i} R_{i}(j)\right)\right]^{2}
$$

Suppose that the minimal desired PSNR (quality) constraint is $P^{m i n}$, e.g. $30 \mathrm{~dB}$, and the maximal satisfied PSNR (quality) constraint is $P^{\max }$, e.g. $45 \mathrm{~dB}$, then $R_{i}^{\min }$ and $R_{i}^{\max }$ can be computed by:

$$
R_{i}^{\text {min }}=\frac{1}{\beta_{i}^{\star}}\left(\frac{P^{\min }}{10 \log _{10} e}-\gamma_{i}^{\star}\right), R_{i}^{\max }=\frac{1}{\beta_{i}^{\star}}\left(\frac{P^{\max }}{10 \log _{10} e}-\gamma_{i}^{\star}\right)
$$

According to Eqn. (11-12), $\gamma_{i}^{\star}, \beta_{i}^{\star}, R_{i}^{\min }$ and $R_{i}^{\max }$ can be obtained and are shown in Table 1. For the parameter $a$, from Section 2.4, we can see that it is related to $a^{0}$, where $a^{0}$ is the variable that satisfies $\sum_{i=1}^{N}\left(\max \left[R_{i}^{\min }, \min \left(\frac{1}{a^{0}}-\frac{\gamma_{i}}{\beta_{i}}, R_{i}^{\max }\right)\right]\right)=R$. Notice that $a^{0}$ be obtained using bisection method as shown in Algorithm 1.

\subsection{Multi-User Rate Allocation}

We compare the proposed method with three approaches: the Absolute Fairness in Rate (AFR), which equally divides the available bandwidth to all the users, the Absolute Fairness in Distortion (AFD), which minimizes the maximal distortion of all the users, and the approach Maximizing the Sum of the PSNRs (MSPSNR). Notice that for AFR, AFD, and MSPSNR, the allocated rate should be within $\left[R_{i}^{\min }, R_{i}^{\max }\right]$. Otherwise, we set it to be $R_{i}^{\min }$ or $R_{i}^{\max }$ and re-allocate the rest rate for other users. 


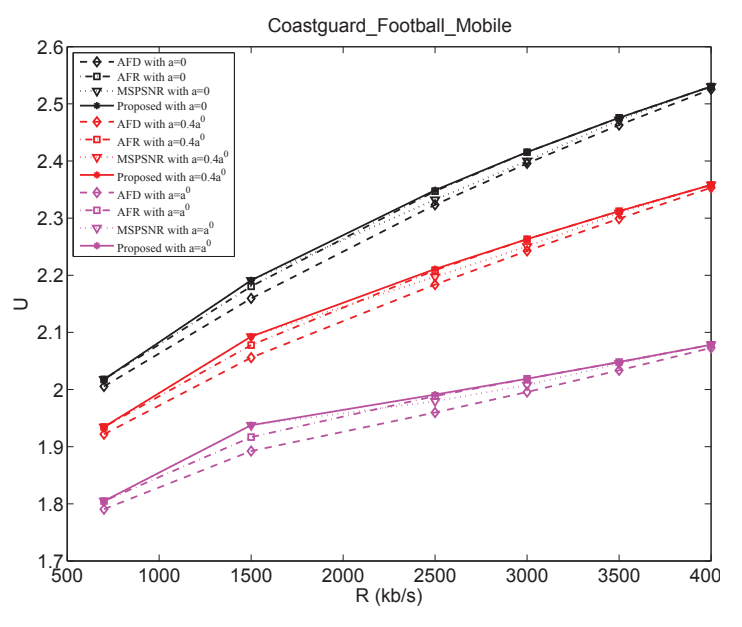

Fig. 3: The sum of natural logarithm utility $U$ vs. the available network bandwidth $R$.

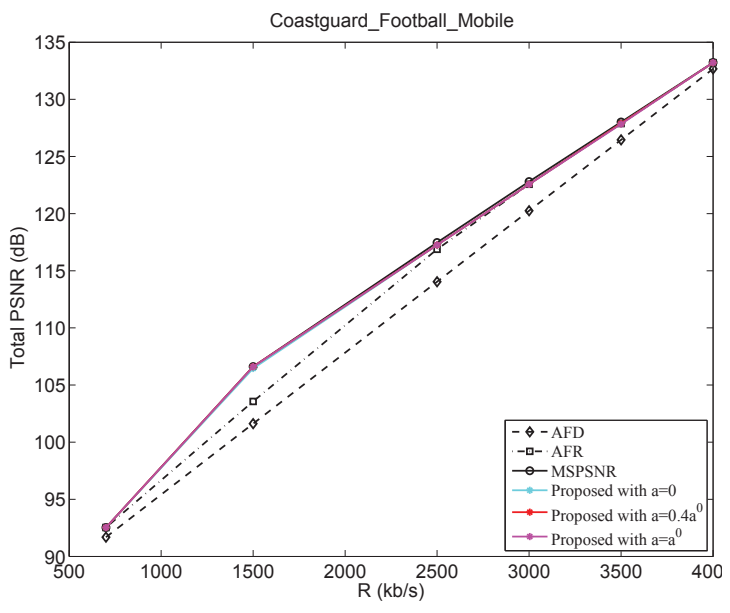

Fig. 4: The sum of natural logarithm utility $U$ vs. the available network bandwidth $R$.

Given the video sequences, $R$, and $a$, the rate allocated to each video sequence can be computed using AFD, AFR, MSPSNR, and the proposed method. Then, setting the allocated bit-rates as target bit-rates, we encode the sequences using H.264 JM 9.0.

We assume that there are three users $u_{1}, u_{2}$, and $u_{3}$. They transmit Coastguard, Football, and Mobile to receivers $r_{1}, r_{2}$, and $r_{3}$, respectively. We test $R$ at 700, 1500, 2500, 3000, 3500 and $4000 \mathrm{~kb} / \mathrm{s}$, and $\frac{a}{a^{0}}$ at $0,0.4$, and 1 . The allocated bit-rate for each video sequence in different situations (i.e. different $R$ and $a$ ) using different methods are shown in Figure 2. From Figure 2, we can see that AFR equally allocates the bandwidth to each user if the allocated bit-rates is within $\left[R_{i}^{\min }, R_{i}^{\max }\right]$. AFD tries to allocate more bit-rates to the video sequence that has more complex motion and/or scene (a smaller $\beta^{\star}$ ) to preserve constant quality among different users. On the contrary, MSPSNR favors the video sequence that has a larger $\beta^{\star}$ since allocating more bit-rates to the sequence with a larger $\beta^{\star}$ leads to an increase in the sum of the PSNRs. However, without considering the fairness issue,

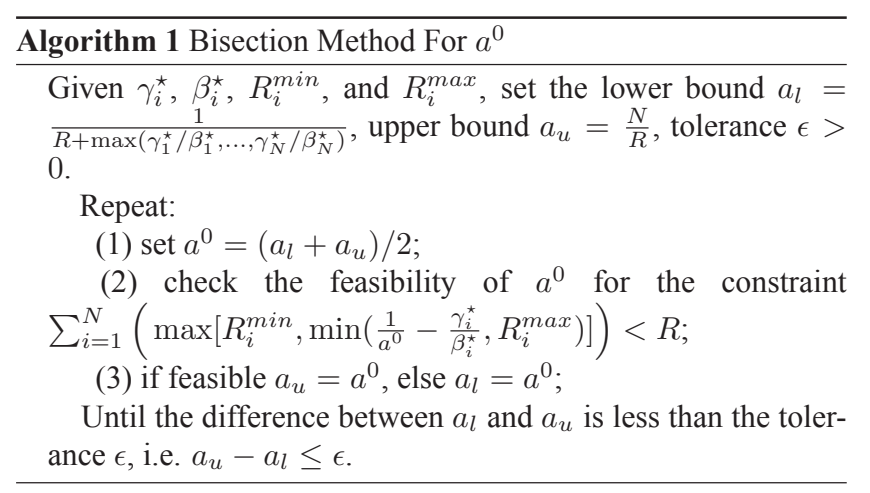

MSPSNR will not allocate more bit-rates than $R_{i}^{\text {min }}$ to the sequence with $\beta_{i}^{\star}$ if there is a sequence with $\beta_{j}^{\star}>\beta_{i}^{\star}$ who has not been allocated its maximal rate requirement $R_{j}^{\max }$ yet. By taking the proportional fairness into account, the proposed method can avoid this disadvantage and keep some balance between the sequences with a larger $\beta^{\star}$ and a smaller $\beta^{\star}$ to achieve fairness among users.

Let $U=\sum_{i=1}^{N} \ln \left(U_{i}\right)$ be the sum of the natural logarithm utility of all the users, and $T_{-} P S N R=\sum_{i=1}^{N} P S N R_{i}$ be the sum of the $P S N R_{i}$ of all the users. In Figure 3 and 4, we show $U$ versus $R$ and $T_{-} P S N R$ versus $R$, respectively. We can see that for all different choices of $a$, the proposed method achieves the highest $U$ at any fixed $R$ with a negligible loss in the system performance (the sum of the PSNRs).

\section{CONCLUSIONS}

In this paper, we proposed a game-theoretic framework for multiuser multimedia rate allocation. Different from the traditional optimization-based approaches, which mainly focused on the efficiency issue, e.g. maximizing the system performance, the proposed method not only considered the efficiency issue but also the fairness issue. From the experimental results on the real video sequences, we could see that with the proportional fairness criterion, the proposed game-theoretic method could efficiently and fairly allocate bit-rates to different users by allocating more bitrates to the sequence with slower motion and/or simpler scene (i.e. a larger $\beta^{\star}$ ) while keeping an eye on the sequence with a smaller $\beta^{\star}$.

\section{REFERENCES}

[1] J. R. Corbera and S. Lei, "Rate control in DCT video coding for low-delay communications," IEEE Trans. Circuits Syst. Video Technol., vol. 9, pp. 172$185,1999$.

[2] C. Shen and M. V. D. Schaar, "Optimal resource allocation for multimedia applications over multiaccess fading channels," IEEE Trans. Wirless Commun. (to appear).

[3] K. Stuhlmuller, N. Farber, M. Link, and B. Girod, "Analysis of video transmission over lossy channels," IEEE J. Sel. Areas Commun., vol. 18, pp. 1012 $1032,2000$.

[4] Yan Chen and Oscar C. Au, "Simultaneous RD-Optimized rate control and video de-noising," in Proc. IEEE Int. Conf. Acoustics, Speech and Signal Processing, 2008.

[5] Zhu Han, Zhu Ji, and K. J. Ray Liu, "Fair multiuser channel allocation for OFDMA networks using Nash bargaining solutions and coalitions," IEEE Trans. Commun., vol. 53, pp. 1366-1376, 2005. 